\title{
Compact calibration source for thermal infrared Fourier-transform spectrometer
}

\begin{abstract}
Alexey Shakun, Fedor Martynovich, Nikolay Ignatiev, Alessandro Maturilli, Aleksandr Santos-Skripko, et al.
\end{abstract}

Alexey Shakun, Fedor Martynovich, Nikolay Ignatiev, Alessandro Maturilli, Aleksandr Santos-Skripko, Vladimir Savosin, Igor Stupin, Gabriele Arnold, Alexei Grigoriev, Oleg Korablev, "Compact calibration source for thermal infrared Fourier-transform spectrometer," Proc. SPIE 11128, Infrared Remote Sensing and Instrumentation XXVII, 1112803 (9 September 2019); doi: $10.1117 / 12.2528692$

SPIE Event: SPIE Optical Engineering + Applications, 2019, San Diego, California, United States 


\title{
Compact calibration source for thermal infrared Fourier-transform spectrometer
}

\author{
Alexey Shakun*a ${ }^{\mathrm{a}}$, Fedor Martynovich ${ }^{\mathrm{a}}$, Nikolay Ignatiev ${ }^{\mathrm{a}}$, Alessandro Maturilli ${ }^{\mathrm{b}}$, Aleksandr Santos- \\ Skripko ${ }^{\mathrm{a}}$, Vladimir Savosin ${ }^{\mathrm{a}}$, Igor Stupin ${ }^{\mathrm{a}}$, Gabriele Arnold ${ }^{\mathrm{b}}$, Alexey Grigoriev ${ }^{\mathrm{a}}$, Oleg Korablev ${ }^{\mathrm{a}}$ \\ ${ }^{a}$ Space Research Institute (IKI), 84/32 Profsoyuznaya, 117997 Moscow, Russia; \\ ${ }^{\mathrm{b}}$ Deutsches Zentrum für Luft- und Raumfahrt e.V. (DLR), Institute of Planetary Research, \\ Rutherfordstrasse 2, 12489 Berlin, Germany. \\ *avshakun@iki.rssi.ru; phone+74953336245
}

\begin{abstract}
Infrared Fourier-transform spectrometers (FTIR) onboard of the planetary missions are commonly used for the thermal sounding of the atmosphere and retrieval of aerosol profiles. To derive a calibrated spectrum of the target source, one needs three separate measurements: the target source itself and two calibration measurements of sources with known emissivity and temperature. An overview of the design of a compact in-built calibration source (a blackbody) emitting at $210-330 \mathrm{~K}$ for a spaceborne FTIR instrument is presented. Mechanically it is an aluminum structure matching the aperture of the instrument. The emissivity depends on its surface relief and finish. Four different types of surface shape are considered. The best-achieved emissivity is better than 0.99 (at $15 \mu \mathrm{m}$ ). The optimal placement of heaters allowing for minimal thermal non-uniformity $(0.1 \mathrm{~K})$ across the aperture is found. The accuracy of the thermal control is also $\sim 0.1$ $\mathrm{K}$. We discuss the thermal control system and its characteristics (accuracy and drift). The proposed design accounts for a minimum mass applicable to the space instrumentation. For a one-inch aperture, the mass is $0.12 \mathrm{~kg}$. The expected accuracy of the instrument calibrated with the designed blackbody is estimated.
\end{abstract}

Keywords: Fourier-transform spectrometer, calibration source, IR remote sensing, Mars atmosphere

\section{INTRODUCTION}

The infrared Fourier-transform spectrometer FAST (Fourier for Atmospheric Species and Temperature) [1] is a part of the ExoMars landing platform science payload [2]. Many instruments of this payload target a long-term study of the near-surface atmosphere of Mars. The primary science goal of FAST is to measure trace gases in the Martian atmosphere observing sun through the atmosphere. Also, FAST aims on long term monitoring of climatological parameters (aerosol and temperature profiles) $[3,4,5$, and 6$]$.

To calibrate the FTIR data, one needs an infrared source with known parameters (emissivity and temperature). The compact infrared calibration source (a blackbody) was developed as a part of the FAST instrument. The thermal uniformity of the blackbody was estimated via modeling for two cases: the emitting surfaces fabricated out of aluminum alloy and copper. The optimal positions of the heaters (regarding the thermal uniformity) were also found trough modeling. Measurements of the emissivity for different surface reliefs and coatings were performed to find the optimal configuration.

The present paper describes an ambient-temperature infrared in-built calibration source developed for the FAST FTIR to be installed on the ExoMars landing platform. The principal technical solutions, results of the measurements, and modeling are presented.

Infrared Remote Sensing and Instrumentation XXVII, edited by Marija Strojnik, Gabriele E. Arnold, Proc. of SPIE Vol. 11128, $1112803 \cdot$ ? 2019 SPIE · CCC code: 0277-786X/19/\$18 · doi: 10.1117/12.2528692 


\section{DESIGN OF THE INFRARED CALIBRATION SOURCE}

Key parameters that have to be considered in the design of the infrared calibration source for FTS are the low thermal gradient of the surface, high accuracy of the blackbody temperature control and its high emissivity. For instruments targeting atmospheric measurements, the critical parameters are the accuracy of the temperature control and the thermal insulation of the calibration source from the FTS. At the Martian surface, protection from the ambient dust becomes an important task, because even a perfect calibration source degrades while it becomes covered by dust, decreasing the accuracy of the instrument calibration.

A 3-D model of the infrared blackbody developed for FAST is shown in Fig. 1. The main element of the infrared calibration source is an aluminum disk with profiled and painted (EKOM-2 enamel by Kompozit, Russia) frontal surface. The key parameters of the FAST blackbody emitting surface profile $\left(\mathrm{h}=5 \mathrm{~mm} ; \mathrm{x}=3.3 \mathrm{~mm} ; \alpha=30^{\circ} ; \beta=10^{\circ}\right)$ are shown in Fig. 2. The blackbody unit is assembled in a polyamide casing covered by MLI (multi-layer insulation) to decrease the heat flow inside the FTS. The infrared calibration source has a mass of $\sim 0.12 \mathrm{~kg}$ and occupies a volume of $60 \times 75 \times 70 \mathrm{~mm}^{3}$. Its clear aperture is $34 \mathrm{~mm}$ that is amply sufficient to fill the entrance aperture of 1-inch FTS for calibration.

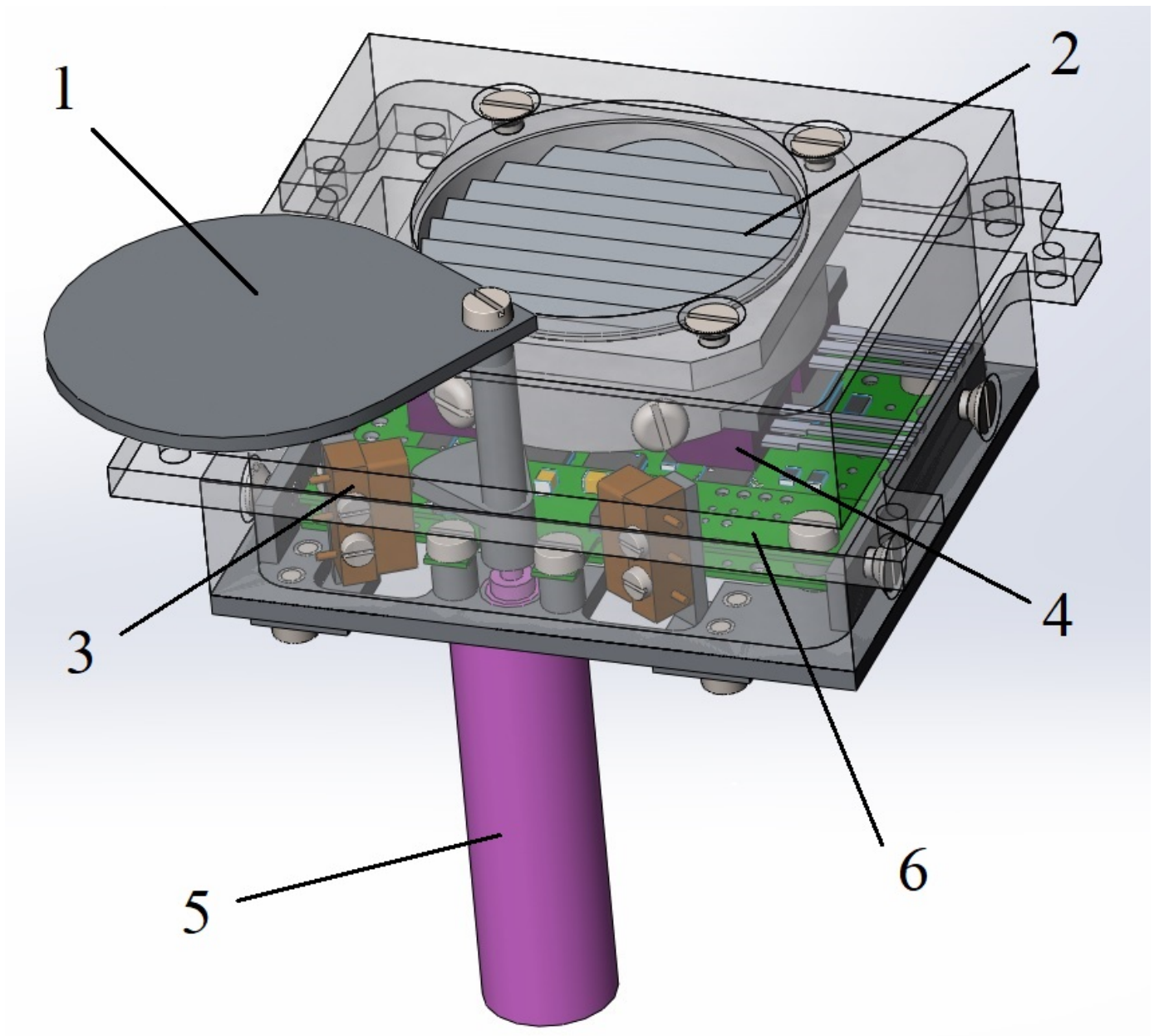

Figure 1. A 3-D model of 1-inch infrared blackbody calibration source. (1) protecting cover; (2) blackbody surface; (3) cover open/closed sensor; (4) heater; (5) cover drive (geared brushed DC motor); (6) blackbody electronics PCB. 


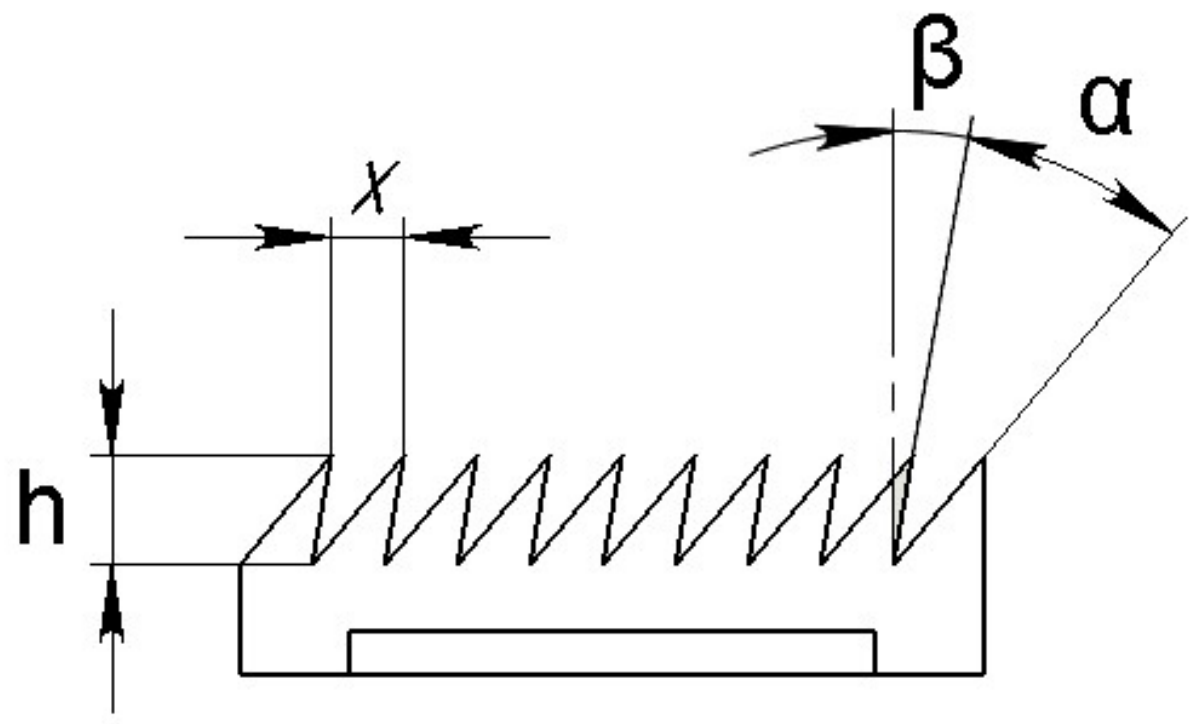

Figure 2. Characteristics of the emitting surface profile of the FAST infrared calibration source.

Four heaters (resistors) are attached to the back side of the item to provide the required temperature. Three platinum thermal sensors (Pt1000 by Honeywell Inc., USA) are glued into holes in the rear side of the device, one of them is included into the thermal regulation loop and two others measure the temperature of the item. A protective cover moved by brushed DC motor (Maxon motor AG, Switzerland) provides dust protection of the profiled painted surface. The cover has two positions (open and closed) controlled by Hall sensors and mechanical switches (for redundancy). The thermal control and protective cover drive is operated by dedicated electronics, occupying one PCB, assembled within the blackbody unit. Power consumption of the heaters is $3 \mathrm{~W}$. Time constant for increasing the temperature of the FAST emitting item on the $20 \mathrm{~K}$ is $\sim 4 \mathrm{~min}$ (for the range of the start temperatures from $230 \mathrm{~K}$ to $300 \mathrm{~K}$ ).

\section{MEASUREMENTS AND MODELING RESULTS}

The thermal uniformity of the FAST blackbody emitting item was modeled to find the optimal heaters position and estimate thermal uniformity of the structure (Fig. 3). For the aluminum alloy device the surface thermal gradient is $0.25 \mathrm{~K}$. However, within the central part of the emitting item with diameter of $28 \mathrm{~mm}$, which is sized to fill the full FAST aperture the gradient remains within $\leq 0.1 \mathrm{~K}$. The same maximal value of the thermal gradient $(\leq 0.1 \mathrm{~K})$ is obtained between the emitting and the rear surfaces of the emitting item. The accuracy of the thermal sensors applied is better than $0.1 \mathrm{~K}$, and the overall accuracy is, therefore, sufficient for the sub-degree thermal sounding of the Martian atmosphere. The use of a copper emitting surface for the same design provides better thermal uniformity of $\sim 0.03 \mathrm{~K}$, but the corresponding increase of mass (plus $30 \mathrm{~g}$ ) appears incompatible with the mass budget of the FAST instrument. 

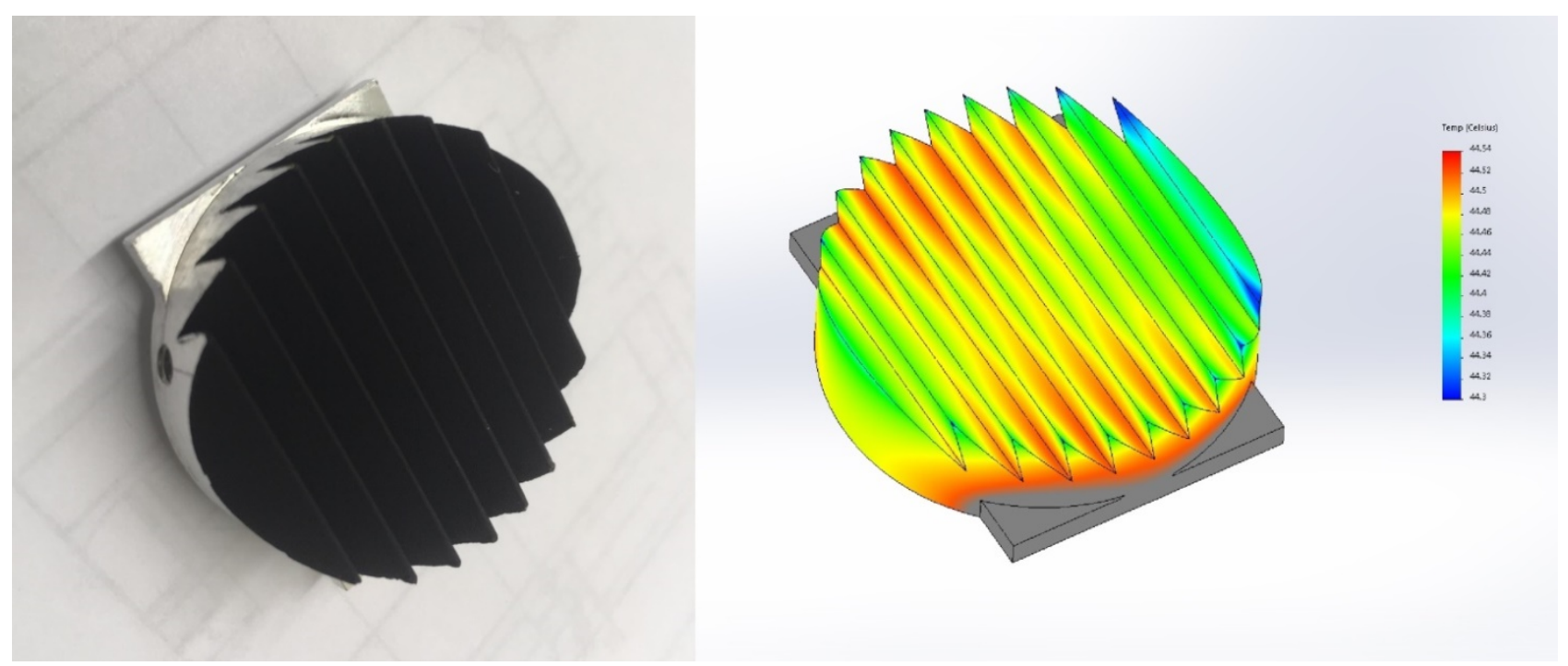

Figure 3. FAST black body (painted mechanical part and its modeled thermal gradient).

The emissivity was calculated from the measured hemispherical reflectance values for different types of the emitting surfaces (Fig. 4) in a wide spectral range $\left(600-5000 \mathrm{~cm}^{-1}\right)$. The reflectance of a flat surface, the calibration source of TIRVIM [7], and the emitting surfaces for FAST of two types (the one described, and the one with shallower relief, $\mathrm{h}=3$ $\mathrm{mm} ; \mathrm{x}=2 \mathrm{~mm} ; \alpha=30^{\circ} ; \beta=10^{\circ}$ ) was measured. All samples were measured at the same ambient temperature and were cowered by the same paint. Higher emissivity values were obtained for the TIRVIM blackbody $(\sim 0.985)$ and for the emitting item of FAST ( 0.99). However, the TIRVIM calibration source is assembled of separate blades [8] and has lower thermal uniformity comparing with the solid body while it is heated.

Hemispherical reflectance measurements are performed at the Planetary Spectroscopy Laboratory (PSL) of the German Aerospace Center (DLR), in Berlin, Germany. The bi-directional reflectance of samples (at room temperature) was measured using Vertex80V spectrometer. High power Globar lamp (24 V, water cooled) was used as a source. The samples were placed in the window of an integrating sphere (with gold-coated surface). Measured spectra were compared to the reference spectrum of the empty sphere to find the hemispherical reflectance.

As a continuation of the present work, measurements of reflectance for different coatings for the same emitting surface are planned. It can result in slightly better emissivity values and fulfill the goal of finding the optimal paint for the specified spectral range. Another possibility to increase the emissivity is optical modeling based on the experimentally found profile of the FAST emitting item. 


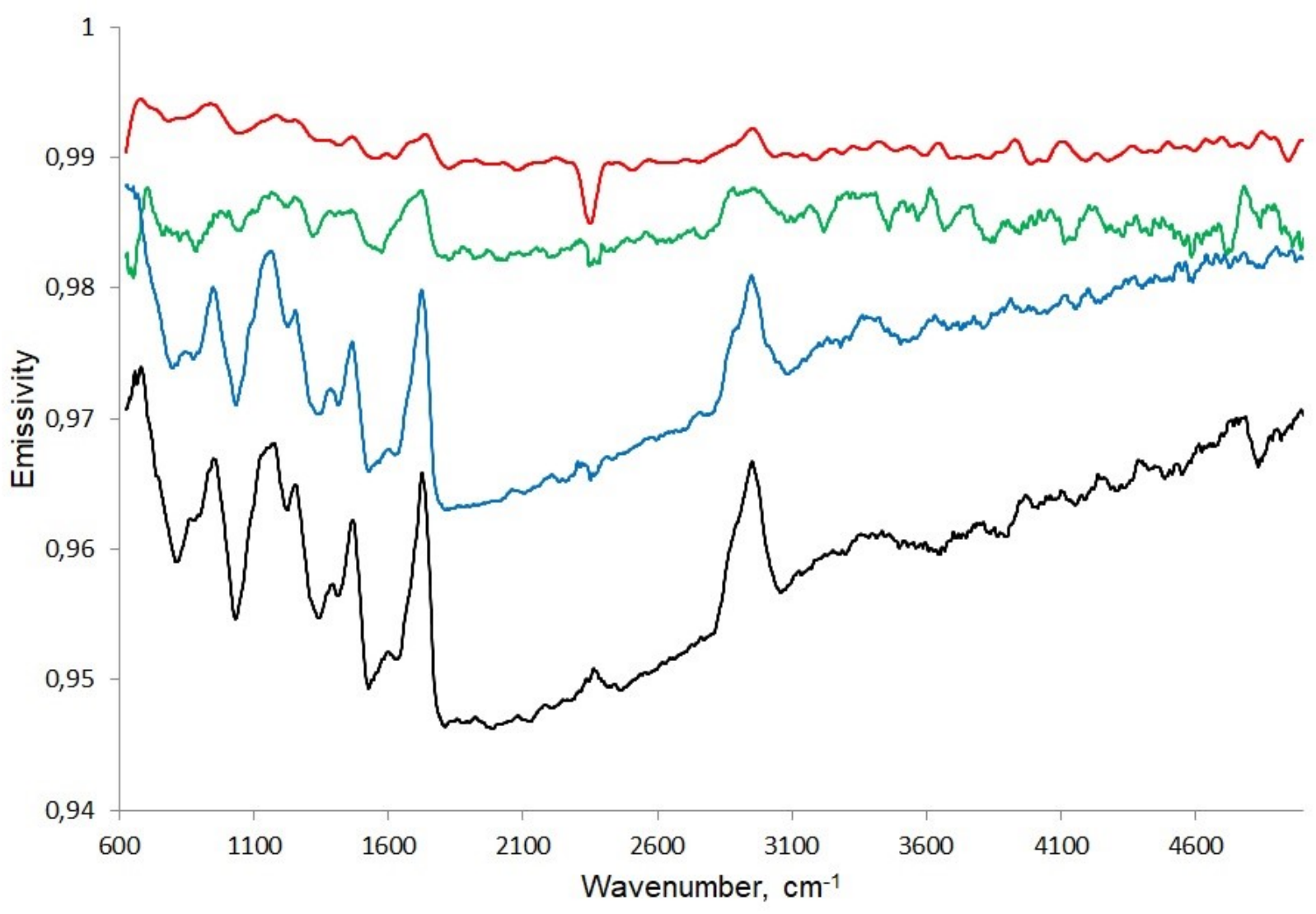

Figure 4. Emissivity spectra measured for different versions of the calibration sources. Black - painted flat surface; blue profiled surface with the smaller grooves; green - the calibration source of the TIRVIM FTIR; red - the FAST emitting item.

\section{CONCLUSIONS}

Experimental and modeling investigation of the critical parameters for the infrared calibration source of the FAST/ExoMars instrument was performed. The temperature of the emitting surface measured with the accuracy $\sim 0.1 \mathrm{~K}$. Its emissivity is $\sim 0.99$ for the wide spectral range $(5-17 \mu \mathrm{m})$ and $\sim 0.993$ for the $15 \mu \mathrm{m}$ band of the $\mathrm{CO}_{2}$. The blackbody unit is equipped with a custom designed cover for the dust protection. Heaters with the power consumption of $3 \mathrm{~W}$ control the temperature of the infrared calibration source within $210-330 \mathrm{~K}$. The case of the unit is made of polyamide and covered by MLI to decrease the heat flow inside the FAST instrument. The characteristics of the infrared calibration source can be further improved by using a copper emitting item, experiments with paints and coatings, and possibly finding more efficient profile parameters.

\section{ACKNOWLEDGEMENTS}

ExoMars is a space mission by ESA and Roscosmos. The development and fabrication of FAST instrument was funded by Roscosmos. Alexey Shakun, Igor Stupin and Oleg Korablev acknowledge support from Russian Science Foundation (RSF grant 16-12-10453), which enabled to perform experiments, computer modeling and laboratory measurements described in sections 2 and 3. 


\section{REFERENCES}

[1] A. Shakun, O. Korablev, B. Moshkin, A. Grigoriev, N. Ignatiev, I. Maslov, O. Sazonov, D. Patsaev, A. Kungurov, A. Santos-Skripko, A. Zharkov, I. Stupin, D. Merzlyakov, V. Makarov, F. Martinovich, Y. Nikolskiy, V. Shashkin "Fourier Transform Spectrometers for remote sensing of planetary atmospheres and surfaces," CEAS Space J. 9(4), 399-409 (2017).

[2] L.M. Zelenyi, O.I. Korablev, D.S. Rodionov, B.S. Novikov , K.I. Marchenkov, O.N. Andreev, E.V. Larionov "Scientific objectives of the scientific equipment of the landing platform of the ExoMars-2020 mission," Solar Syst. Res., 49(7), 509-517 (2015).

[3] Hanel, R., Conrath, B., Hovis, W., Kunde, V., Lowman, P., Maguire, W., Pearl, J., Pirraglia, J., Prabhakara, C., and Schlachman, B., "Investigation of the Martian environment by infrared spectroscopy on Mariner 9," Icarus 17, 423442 (1972).

[4] Grassi, D., Ignatiev, N. I., Zasova, L. V.; Maturilli, A., Formisano, V., Bianchini, G. A., Giuranna, M. "Methods for the analysis of data from the Planetary Fourier Spectrometer on the Mars Express Mission," Planet. Space. Sci. 53(10), 1017-1034 (2005).

[5] Smith, M. D., J. C. Pearl, B. J. Conrath and P. R. Christensen, "One Martian year of atmospheric observations by the thermal emission spectrometer," Geophysical Research Letters 28, 4263-4266 (2001).

[6] Smith, M. D., "Interannual variability in TES atmospheric observations of Mars during 1999-2003," Icarus 167, 148165 (2004).

[7] O. Korablev, F. Montmessin, A. Trokhimovskiy, A.A. Fedorova, A.V. Shakun, A.V. Grigoriev, B.E. Moshkin, N.I. Ignatiev, et al., "The Atmospheric Chemistry Suite (ACS) of three spectrometers for the ExoMars 2016 Trace Gas Orbiter," Space Sci Rev 214(7), doi: 10.1007/s11214-017-0437-6 (2018).

[8] B.E. Moshkin, S.V. Maximenko, A.A. Balashov, V.A. Vagin, M.A. Shilov "Technological black body for infrared spectral range," Instrum Exp Tech 58(5), 154-155 (2010). 